\title{
LAPAROSCOPIC MANAGEMENT OF URETERAL ENDOMETRIOSIS
}

\author{
Nicolae BACALBASA ${ }^{1,2} \bowtie$, Irina BALESCU ${ }^{3}$, Mihaela VILCU ${ }^{1,2}$, Iulian BREZEAN ${ }^{1,2}$ \\ ${ }^{1}$ „Carol Davila“ University of Medicine and Pharmacy, Bucharest, Romania \\ 2 „Ion Cantacuzino“ Clinical Hospital, Bucharest, Romania \\ ${ }^{3}$ Ponderas Academic Hospital, Bucharest, Romania
}

Received 26 Aug 2019, Accepted 07 Oct 2019

hitps://doi.org/10.31688/ABMU.2019.54.4.17

\begin{abstract}
Ureteral endometriosis is characterized by the development of deep infiltrating islets of endometrial tissue affecting the ureter, the optimal management in such cases being still poorly defined. Once the techniques of laparoscopic surgery improved, this minimally invasive approach has been proposed with encouraging results in treating such cases. When it comes to the types of surgical procedure which are needed in such cases, they are chosen accordingly to the degree, extent and localization of the endometrial tissue. This is a literature review of the largest studies which were conducted on the theme of the laparoscopic management of ureteral endometriosis; the main analyzed parameters refer to the efficacy and safety of the procedure.
\end{abstract}

Keywords: ureteral endometriosis, resection, laparoscopy.

\section{Résumé}

Approche laparoscopique de l'endométriose urétérale

L'endométriose urétérale est caractérisée par le développement d'îlots infiltrants profonds du tissu endométrial affectant l'uretère, la gestion optimale dans de tels cas étant encore mal définie. Une fois que les techniques de chirurgie laparoscopique se sont améliorées, cette approche peu invasive a été proposée avec des résultats encourageants dans le traitement de tels cas. En ce qui concerne les types d'interventions chirurgicales nécessaires dans de tels cas, ils sont choisis en fonction du degré, de l'étendue et de la localisation du tissu endométrial. Il s'agit d'une revue de la littérature des plus grandes études menées sur le thème de la gestion laparoscopique de l'endométriose urétérale; les principaux paramètres analysés se réfèrent à l'efficacité et à la sécurité de la procédure.

Mots-clés: endométriose urétérale, résection, coelioscopie. 


\section{INTRODUCTION}

Defined by the presence of ectopic islands of endometrial cells and stroma outside the uterine body, endometriosis can be classified according to its depth in superficial - invading the peritoneum, and the ovary or deep infiltrating - invading the viscera such as rectum, colon, urinary tract or the enteral loops by at least $5 \mathrm{~mm}$ in depth. According to the depth of invasion, the clinical signs and symptoms may range between diffuse pelvic pain, constipation, hematuria and complete bowel or urinary tract obstruction ${ }^{1-3}$.

\section{Urinary tract involvement in patients with endo- metriosis}

When it comes to the urinary tract involvement, the most commonly affected organs are represented by the urinary bladder, followed by ureter and kidney, accounting for $0.3-12 \%$ of all cases diagnosed with this pathology $y^{4,5}$. Depending on the degree of invasion, ureteral endometriosis can be classified as extrinsic - if the periureteral tissues or the serosa are involved - and intrinsic, if the muscularis propria or the mucosa is damaged ${ }^{6}$. In cases presenting extrinsic endometriosis, the lesions originate from the peritoneum, ovaries or uterosacral ligaments and produce an extrinsic compression of the ureter, while in intrinsic lesions, invasion of the muscularis or mucosa occurs. However, it seems that extrinsic lesions are up to four times more frequent when compared to intrinsic lesions ${ }^{7,8}$.

In order to rule out which are the predictive preoperative factors regarding the depth of ureteral invasion, Gennaro et al. conducted a study on 82 women in whom ureteral involvement due to endometriosis was encountered ${ }^{9}$. Among these cases the authors identified 15 cases in which deep infiltrating endometriosis was present and in which ureteral resections were needed, and 67 cases in which the urological procedure was limited to ureterolysis. The authors underlined the fact that the most commonly encountered symptom was represented by the abdominal pain, equally encountered between the two groups, while other signs or symptoms, such as urinary urgency, hematuria, dysuria or hydronephrosis were significantly more common among patients presenting deep infiltrating lesions?

As for the preoperative investigations which might predict the presence of ureteral involvement, it has been widely accepted that pelvic MRI, cystoscopy and urography might give important information in regard to the extent of the disease. More recently, a study conducted by Lima et al. demonstrated that the presence of endometrial nodules at the level of the uterosacral ligament represents a strong predictive factor for the association of ureteral involvement. Therefore, they concluded that the presence of uterosacral nodules measuring at least $1.75 \mathrm{~cm}$ on the right side and $1.95 \mathrm{~cm}$ on the left side represents a strong predictive factor for the concomitance of ureteral involvement ${ }^{10}$.

\section{Surgical goals in patients diagnosed with uret- eral endometriosis}

Once the diagnosis of ureteral endometriosis is established, surgery is needed in order to relieve the ureteral obstruction, to preserve the renal function and to prevent the development of any recurrent disease $^{4}$. In order to achieve these goals certain authors proposed initially performing a limited procedure such as ureterolysis; however, in a significant number of cases, recurrent disease might be encountered after a relatively short period of time; therefore, in such cases resection and urinary tract reconstruction is finally the option of choice. For example, in Ghezzi's study which included 33 patients who were submitted to laparoscopic ureterolysis, the rate of early recurrence imposing reoperation reached 12\% during the first three months postoperatively ${ }^{11}$. In the last decades, once the techniques of minimally invasive surgical approach improved, the number of cases submitted to this approach increased as well; therefore, a higher number of such cases have benefited lately from the advantages of the laparoscopic approach.

\section{Studies investigating the role of laparoscopic ureteral resection for deep infiltrating endome- triosis}

The utility of the laparoscopic approach in such cases has been established in the last decades once the minimally invasive approach started replacing the conventional approach; therefore, it has been widely accepted that the minimally invasive approach might offer certain advantages, such as a magnification of the view and better individualization of the structures. In the study conducted by Chudzinski et al and published in 2017 the authors included 17 patients presenting ureteral involvement in which ureteral resection and reimplantation were needed ${ }^{12}$. Among these cases, the ureteral involvement was known preoperatively in $82 \%$ of cases, in $23 \%$ of them bilateral ureteral involvement being reported; moreover $35 \%$ of cases also associated renal atrophy, while renal function impairment was found in $23 \%$ of cases. When it comes to the preferred approach, the authors underlined the fact that laparotomy was the option of choice in $41 \%$ of cases, being followed by laparoscopy in 35\% of cases and robotics in $23 \%$ of cases; however, the rate of laparotomies decreased from $63 \%$ before the year of 2010 to $23 \%$ after the year of 2010. Among cases submitted to a minimally invasive approach, there were three cases in which 
conversion to open surgery was needed due to the presence of bowel injury - in one case and due to the presence of iatrogenic ureteral lesions - in two cases $^{12}$. In a similar study conducted by Sconman et al in Tel-Aviv, Israel, and which included seven patients, the authors reported the necessity to convert from laparoscopy to laparotomy in two cases, while in other four cases laparotomy was the option of choice $^{13}$; interestingly, in all cases a previous history of at least one surgical intervention for endometriosis was reported. In all cases resection with ureteral reimplantation by using a psoas hitch technique was the preferred technique. After a mean follow-up period of $42+/ 20$ months, a single patient reported no improvement of the symptoms ${ }^{13}$.

The benefits of the laparoscopic approach in patients with ureteral endometriosis were also demonstrated by Miranda-Mendoza in 2012 $2^{14}$. The study included 13 patients submitted to surgery in a minimally invasive manner, six cases being submitted to ureteral resections, while the remaining seven cases being submitted to ureterolysis. The histopathological studies of the resected specimens demonstrated the presence of ureteral invasion in all cases in which segmental ureteral resections were performed. However, at that moment the rates of postoperative complications remained high, three of the 13 cases developing vesical-vaginal leaks. However, after a median follow-up period of 24 months all but one cases reported a significant improvement of the symptoms, as well as the absence of recurrent disease. In the $13^{\text {th }}$ case the patient developed an obstructive uropathy and an ureteroneocystostomy was performed ${ }^{14}$.

One of the largest studies which investigated the feasibility and safety of laparoscopic management of ureteral endometriosis was the one conducted by Cavaco-Gomes et al and published in $2017^{2}$. The study, a review which included 18 articles published since 1997 and 700 patients demonstrated the efficacy of the method and underlined the fact that ureteral endometriosis is a more common finding at the level of the left ureter (being encountered in 53.6\%), followed by the right ureter (in $35.8 \%$ of cases) and bilateral lesions in only $10.6 \%$ of cases). As for the type of surgical procedure which was performed, it consisted of ureterolysis alone in 579 cases and ureteral resection in the remaining ones; moreover, in two cases nephrectomy was imposed by the secondary renal atrophy, while in other $19.8 \%$ of cases concomitant urinary bladder resection was also needed. Other common resections were represented by rectovaginal or uterosacral ones. When it comes to the short-term outcomes, the most frequently reported complications were represented by ureteral leaks or stenosis (in 14 cases), hemorrhagic events (in three cases), anastomotic digestive leaks (in two cases), bowel perforation (in one case), bladder atony (in one case) and vesicovaginal leak (in one case). As for the long-term outcomes, $90.5 \%$ of cases reported the resolution or improvement of the symptoms. The authors also underlined the recurrent pattern of this pathology as well as the fact that most often this disease is a multicentric one ${ }^{2}$.

In a recent study conducted by Ceccaromi et al, the authors included 160 patients submitted to total laparoscopic ureteroneocystostomy between January 2009 and December 2016 ${ }^{15}$; in all cases surgery was successfully ended in a minimally invasive manner, while the histopathological studies confirmed the presence of ureteral invasion in all cases (in $45.6 \%$ the urinary tract involvement occurred in an extrinsic manner while in the remaining $54.4 \%$ of cases an extrinsic manner being demonstrated); after resection the authors reported the use of the psoas technique of reconstruction in $58.7 \%$. Moreover, concomitant digestive tract invasion was seen in $75.6 \%$ and imposed performing a bowel resection. When it comes to the short-term outcomes, the authors reported the necessity of reoperation in $4.4 \%$ of cases; as for the long-term outcomes, after a follow-up of six months, $15 \%$ of cases reported impaired bladder voiding. In the meantime after a mean follow-up period of 20.5 months regression of the symptoms was seen in most patients, only $1.2 \%$ of them requiring a ureteroneocystostomy on the opposite side ${ }^{15}$.

When it comes to the modalities of reconstruction after ureteral resections, while certain authors prefer performing an uretero-ureteral anastomosis whenever is possible, other authors consider that a ureteral reimplantation in the urinary bladder through an ureteroneocystostomy should be the option of choice due to a lower risk of complications, such as anastomotic stenosis ${ }^{14,16,17}$.

Moreover, in cases presenting deep infiltrating lesions, a combined laparoscopic and cystoscopic approach might be tempted, encouraging results being reported so far ${ }^{18,19}$. This combined approach seems to have the benefit of removing only the affected segment of the detrusor muscle decreasing in this way the risk of postoperative complications such as dysuria, polakiuria or bladder denervation. The procedure begins in a laparoscopic manner by identifying and dissecting the endometrial nodule followed by a cystoscopic step which identifies the area of mucosal involvement ${ }^{20,21}$.

\section{Conclusions}

Although urinary tract involvement is a common finding in patients diagnosed with endometriosis, ureteral invasion is lower when compared to the 
rates of urinary bladder invasion. Even though, whenever ureteral involvement is discovered, laparoscopy seems to be a feasible method in order to achieve an adequate alleviation of the symptoms. Depending on the degree of invasion and on the site of invasion multiple therapeutic strategies might be proposed, ranging from ureterolysis to ureteral resection with end to end anastomosis or ureteral resection followed by ureteral reimplantation by ureteroneocystostomy. Although initially the rates of postoperative complications were considerable, once more experience has been gained these rates of morbidity decreased. In the meantime, the rates of recurrences seen after a long-term follow-up seem to report a descendent trend during the last decade.

\section{Acknowledgements}

This work was supported by the project entitled „Multidisciplinary Consortium for Supporting the Research Skills in Diagnosing, Treating and Identifying Predictive Factors of Malignant Gynecologic Disorders“, project number PN-III-P11.2-PCCDI2017-0833.

\section{Compliance with Ethics Requirements:}

„The authors declare no conflict of interest regarding this article"

"The authors declare that all the procedures and experiments of this study respect the ethical standards in the Helsinki Declaration of 1975, as revised in 2008(5), as well as the national law."

\section{References}

1. Koninckx PR, Martin DC. Deep endometriosis: a consequence of infiltration or retraction or possibly adenomyosis externa? Fertil Steril 1992; 58(5): 924-928.

2. Cavaco-Gomes J, Martinho M, Gilabert-Aguilar J, Gilabert-Estelles J. Laparoscopic management of ureteral endometriosis: A systematic review. Eur J Obstet Gynecol Reprod Biol 2017; 210: 94-101.

3. Fritz MA, Speroff L. Clinical Gynecologic Endocrinology and Infertility. 8 ed. Philadelphia: Lippincott Williams \& Wilkins; 2011. p. 1221-1223.

4. Maccagnano C, Pellucchi F, Rocchini L et al. Ureteral endometriosis: proposal for a diagnostic and therapeutic algorithm with a review of the literature. Urol Int 2013; 91(1): 1-9.
5. Sillou S, Poiree S, Millischer AE, Chapron C, Helenon $O$. Urinary endometriosis: MR imaging appearance with surgical and histological correlations. Diagn Interv Imaging 2015;96(4):373-381.

6. Yohannes P. Ureteral endometriosis. J Urol 2003;170(1): 20-25.

7. Bulkley GJ, Carrow LA, Estensen RD. Endometriosis of the ureter. J Urol 1965;93: 139-143.

8. Fujita K. Endometriosis of the ureter. J Urol 1976;116(5):664.

9. Gennaro KH, Gordetsky J, Rais-Bahrami S, Selph JP. Ureteral endometriosis: preoperative risk factors predicting extensive urologic surgical intervention. Urology 2017;100: 228-233.

10. Lima R, Abdalla-Ribeiro H, Nicola AL, Eras A, Lobao A, Ribeiro PA. Endometriosis on the uterosacral ligament: a marker of ureteral involvement. Fertil Steril 2017;107(6):1348-1354.

11. Ghezzi F, Cromi A, Bergamini V, Serati M, Sacco A, Mueller MD. Outcome of laparoscopic ureterolysis for ureteral endometriosis. Fertil Steril 2006;86(2):418-422.

12. Chudzinski A, Collinet P, Flamand V, Rubod C. Ureterovesical reimplantation for ureteral deep infiltrating endometriosis: A retrospective study. J Gynecol Obstet Hum Reprod 2017:46(3):229-233.

13. Schonman R, Dotan Z, Weintraub AY, et al. Long-term follow-up after ureteral reimplantation in patients with severe deep infiltrating endometriosis. Eur J Obstet Gynecol Reprod Biol 2013;171(1):146-149.

14. Miranda-Mendoza I, Kovoor E, Nassif J, Ferreira H, Wattiez A. Laparoscopic surgery for severe ureteric endometriosis. Eur J Obstet Gynecol Reprod Biol 2012;165(2):275-279.

15. Ceccaroni M, Ceccarello M, Caleffi G, et al. Total laparoscopic ureteroneocystostomy for ureteral endometriosis: a single-center experience of 160 consecutive patients. J Minim Invasive Gynecol 2019; 26(1): 78-86.

16. Bratu OG, Cherciu AI, Bumbu A, et al. Retroperitoneal tumors - treatment and prognosis of tumor recurrence. Rev Chim (Bucharest) 2019;70(1):191-194.

17. Bodean $\mathrm{O}$, Bratu $\mathrm{O}$, Munteanu $\mathrm{O}$, et al. Iatrogenic injury of the low urinary tract in women undergoing pelvic surgical interventions. Arch Balk Med Union 2018;53(2):281-284.

18. Tiglis M, Neagu TP, Elfara M, et al. Nefopam and its role in modulating acute and chronic pain. Rev Chim (Bucharest) 2018;69(10):2877-2880.

19. Diaconu CC, Arsene D, Balaceanu A, Bartos D. A rare tumor revealed by abdominal trauma: case presentation. Romanian Journal of Morphology and Embryology 2014;55(3):973-976.

20. Millochau JC, Stochino-Loi E, Defortescu G, Darwish B, Roman H. Combined laparoscopic and cystoscopic approach in large deep infiltrating endometriosis of the bladder. J Gynecol Obstet Hum Reprod 2017;46(9):691-692.

21. Bodean O, Bratu OG, Bohiltea R, et al. The efficacy of synthetic oral progestin pills in patients with severe endometriosis. Rev Chim (Bucharest) 2018;69(6):1411-1415. 